\title{
Polarization Simultons in Stimulated Raman Scattering by Polaritons
}

\author{
Vladimir Feshchenko1, Galina Feshchenko² \\ ${ }^{1}$ Dawson College, Montreal, Canada \\ ${ }^{2}$ Vanier College, Montreal, Canada \\ Email: vfeshchenko@dawsoncollege.qc.ca, feshcheg@vaniercollege.qc.ca
}

How to cite this paper: Feshchenko, V. and Feshchenko, G. (2021) Polarization Simultons in Stimulated Raman Scattering by Polaritons. Journal of Applied Mathematics and Physics, 9, 2193-2204.

https://doi.org/10.4236/jamp.2021.99139

Received: July 20, 2021

Accepted: September 6, 2021

Published: September 9, 2021

Copyright (c) 2021 by author(s) and Scientific Research Publishing Inc. This work is licensed under the Creative Commons Attribution International License (CC BY 4.0).

http://creativecommons.org/licenses/by/4.0/

\begin{abstract}
Expressions are obtained for the shortened Maxwell's equations simulating the evolution of the ultrashort pulses propagating in anisotropic dipole-active crystals in stimulated Raman scattering (SRS) by polaritons. The developed theory considers the case of cubic crystals which become anisotropic due to the deformation of the dielectric constant by the linearly polarized pump wave. The pump field is approximated by a linearly polarized plane electromagnetic wave. The possibility of simultaneous propagation of pulses on both different frequencies (pump and Stokes) and different polarization (simultons) is theoretically shown. It is also shown that the expression for the gain factor $g$ in SRS is consistent with the experimental results for the spectra of ZnS.
\end{abstract}

\section{Keywords}

Nonstationary Stimulated Raman Scattering, Polaritons, Phonons, Solitons, Polarization

\section{Introduction}

In general, the pulses (including solitons) are randomly polarized. However, it is desirable to have pulses with a certain (better yet, predominantly defined polarization). It stands to a reason that the polarization could also be a carrier of the information about processes that took place during the interaction. Especially it is important in the case of optical fibers [1]. One of the features of the propagation of solitons in the randomly birefringent optical fibers is that they are randomly polarized whereas there is a need for the solitons with well-defined polarization. In [1], it was both theoretically and experimentally shown that in special cases when circular birefringence was induced when the fibers were twisted, 
the random linear birefringence was mitigated. In [2], it was shown that an induced process of modulational instability may be exploited for the generation of $\mathrm{THz}$ train of vector dark solitons. The technique of frequency-resolved optical gating was used to completely characterize the intensity and phase of the dark solitons. In the above article, the authors analyzed the experiments that showed that with dual-frequency orthogonal polarization, one may achieve the simultaneous suppression of modulational instability. The second nonlinear phenomenon that is significant for the generation of ultrashort optical pulses is stimulated Raman scattering (SRS). In [3], by using a nanosecond laser as the pump source, polarization modulation instability (PMI) and SRS were experimentally investigated in an optical fiber. It was shown that such investigation on the PMI gain in the As2S5 optical fiber may provide a reference for the development of fiber amplifiers and fiber lasers. The detailed analysis of the polarization of the solitons generated by PMI was provided in [4] in which was found that the circular polarization of the pump pulse in the twisted fiber produces circularly polarized solitons with a high grade of polarization whereas in the fiber without twisting, the soliton polarization was random. The calculation of a polarization-dependent effective SRS gain factor considering the random birefringence character of the fiber and the relative mismatch between the continuous pump and the signal was carried out in [5]. There were also achievements in the theory of solitons and SRS [6]. The numerical simulation of the transient stimulated Raman scattering based on using the inverse scattering transform technique is reported in [7]. A new type of solitons-dissipative Raman solitons-was found based on the nonlinear Ginzburg-Landau equation in [8]. In past years, there was also significant progress related to the development of the micro-resonators which convert laser light into a series of ultrashort pulses traveling around the resonator's circumference [9]. These pulses can propagate while maintaining their shape, therefore, becoming solitons. One of the promising applications of solitons is, of course, spectroscopy. The comparative investigation of characteristics of spontaneous and stimulated Raman scattering (SRS) in different alkali-earth tungstate and molybdate crystals at both high and low-frequency anionic group vibrations was presented, for example, in [10]. It has been found that, among these crystals, the $\mathrm{SrMoO}_{4}$ and $\mathrm{SrWO}_{4}$ crystals are the most perspectives for SRS generation on both stretching and bending modes of internal anionic group vibrations with the strongest SRS pulse shortening under synchronous laser pumping. The significant progress in applying the methods of SRS and CARS (Coherent Anti-Stokes Raman Spectroscopy) in recent years was achieved in biology and medicine [11]-[17]. For example, in [11] it was reported a new procedure for alignment-free frequency modulation SRS utilizing polarization encoding since the SRS microscopy is a powerful method for imaging molecular distributions based on their intrinsic vibrational contrast. The successful application of polarization-sensitive SRS imaging in treating skin, nail, and fungal infections was reported in [18]. 
However, in our opinion, there is a need for studying the processes of the generation of solitons in nonlinear processes with predetermined polarization that would allow generating the stable ultrashort pulses traveling through the medium not only at different frequencies but different polarization (for example, the soliton at one frequency but with two perpendicular polarizations). Such creation of ultrashort stable pulses could significantly increase the resolution of SRS microscopy of imaging of the molecular vibrations. In this paper, we theoretically considered the formation of solitons of such type in transient SRS by polaritons [19]-[24]. Also, in our article principal attention was paid to a calculation and analysis of the gain of both stationary SRS and spontaneous Raman scattering. We considered the case of nonstationary SRS in cubic crystals and showed that the theory developed is consistent with experimental results.

\section{Basic Principles and Equations}

In this paper, we carry out our analysis assuming that the pump field is a linearly polarized plane electromagnetic wave. It is also assumed that the nonlinear medium takes the form of a layer bounded by the planes $z=0$ and $z=L$. The pump wave

$$
\boldsymbol{E}_{l}(\boldsymbol{r}, t)=\boldsymbol{e}_{l} A_{l} \exp \left[i\left(k_{l}^{z} z-\omega_{l} t\right)\right]+c . c .
$$

propagates along the $z$-axis. The subscripts $l, s$, and $p$ henceforth denote the pump (laser), Stokes, and polariton wave fields; $\omega$ are the frequencies, $n$ and $\boldsymbol{k}$ are the refractive indices and the wave vectors in the unpumped medium, and $\boldsymbol{e}$ are the real unit vectors of electromagnetic fields. The medium is nonmagnetic and transparent at frequencies $\omega_{l, s}$. We use the Stokes and polariton fields in the form

$$
\begin{aligned}
& \boldsymbol{E}_{s}(\boldsymbol{r}, t)=\sum_{\mu=1,2} \boldsymbol{e}_{s}^{(\mu)} A_{s}^{(\mu)} \exp \left[i\left(\boldsymbol{k}_{s} \cdot \boldsymbol{r}-\omega_{s} t\right)\right]+c . c . \\
& \boldsymbol{E}_{p}(\boldsymbol{r}, t)=\sum_{\sigma=1,2,3} \boldsymbol{e}_{p}^{(\sigma)} A_{p}^{(\sigma)} \exp \left[i\left(\boldsymbol{W} \cdot \boldsymbol{r}-\omega_{p} t\right)\right]+c . c .
\end{aligned}
$$

where: $\boldsymbol{e}_{s}^{(\mu)} \perp \boldsymbol{k}_{s}, \quad \boldsymbol{e}_{s}^{(1)} \perp \boldsymbol{e}_{s}^{(2)}, \quad k_{s}=q_{s} n_{s}, \quad q_{s}=\omega_{s} / c, \quad \boldsymbol{W}=\boldsymbol{k}_{l}-\boldsymbol{k}_{s}, \quad \boldsymbol{e}_{p}^{(1,2)} \perp \boldsymbol{W}$, $\boldsymbol{e}_{p}^{(1)} \perp \boldsymbol{e}_{p}^{(2)}, \boldsymbol{e}_{p}^{(3)}=\frac{\boldsymbol{W}}{W}, \omega_{p}=\omega_{l}-\omega_{s}$.

The longitudinal component of the Stokes wave can be neglected, but this cannot be done for the polariton wave in the phonon region. It has been shown in [25] that with a further advance into this region all three amplitudes $A_{p}^{(\sigma)}$ first become comparable, after which $A_{p}^{(3)}$ becomes dominant, provided, of course, the excitation of the longitudinal waves is allowed by the selection rules. The phase shift of the polariton wave is determined by the vector $\boldsymbol{W}$ and not by $\boldsymbol{k}_{p} \quad\left(k_{p}=q_{p} \sqrt{\varepsilon_{p}}, q_{p}=\omega_{p} / c, \varepsilon_{p}=\varepsilon_{p}^{\prime}+i \varepsilon_{p}^{\prime \prime}\right.$ is the dielectric constant at the frequency $\omega_{p}$ ).

The fields $\boldsymbol{E}_{s, p}$ are interrelated via the nonlinear part of the polarization $\boldsymbol{P}(\boldsymbol{r}, t)$. The latter quantity has at the frequencies $\omega_{l, s, p}$ the following forms 


$$
\begin{aligned}
P_{l}= & \chi_{l}^{\mu \sigma} A_{s}^{(\mu)} A_{p}^{(\sigma)} \exp \left[i\left(\left(k_{s}^{z}+k_{p}^{(\sigma) z}\right) z-\omega_{l} t\right)\right] \\
& +\gamma_{l}^{\mu \mu^{\prime}} A_{s}^{(\mu)} A_{s}^{\left(\mu^{\prime}\right)^{*}} A_{l} \exp \left[i\left(k_{l}^{z} z-\omega_{l} t\right)\right]+c . c . \\
P_{s}= & \chi_{s}^{\mu \sigma} A_{l} A_{p}^{(\sigma) *} \exp \left[i\left(k_{l}^{z}-k_{p}^{(\sigma) z}\right) z-\omega_{s} t\right] \\
& +\gamma_{s}^{\mu \mu^{\prime}}\left|A_{l}\right|^{2} A_{s}^{\left(\mu^{\prime}\right)} \exp \left[i\left(k_{s}^{z} z-\omega_{s} t\right)\right]+c . c . \\
P_{p}= & \chi_{p}^{\mu \sigma} A_{l} A_{s}^{(\mu) *} \exp \left[i\left(W^{z} z-\omega_{p} t\right)\right]+c . c .
\end{aligned}
$$

where $\quad \chi_{l}^{\mu \sigma}=e_{l}^{i} e_{s}^{(\mu) j} e_{p}^{(\sigma) k} \chi_{l, i j k}\left(\omega_{s}, \omega_{p}\right), \quad \gamma_{l}^{\mu \mu^{\prime}}=e_{l}^{i} e_{s}^{(\mu) j} e_{s}^{\left(\mu^{\prime}\right) j} e_{l}^{m} \gamma_{l, i j k m}\left(\omega_{l}, \omega_{s},-\omega_{s}\right)$, $\chi_{s}^{\mu \sigma}=e_{s}^{(\mu) i} e_{l}^{j} e_{p}^{(\sigma) k} \chi_{s, i j k}\left(\omega_{l},-\omega_{p}\right), \gamma_{s}^{\mu \mu^{\prime}}=e_{s}^{(\mu) i} e_{s}^{(\mu) j} e_{l}^{k} e_{l}^{m} \gamma_{s, i j k m}\left(\omega_{p}, \omega_{l},-\omega_{l}\right)$.

The shortened equations for the amplitudes $A_{l, s, p}^{(\mu, \sigma)}$ are obtained from Maxwell's equations by the standard procedure [26] and take the form

$$
\begin{gathered}
\frac{\partial A_{l}}{\partial z}+\frac{1}{v_{l}^{z}} \frac{\partial A_{s}}{\partial t}=i \frac{2 \pi \omega_{l}}{c n_{l} \cos \theta_{l}^{z}}\left(\chi_{l}^{\mu \sigma} A_{s}^{(\mu)} A_{p}^{(\sigma)}+\gamma_{l}^{\mu \mu^{\prime}} A_{s}^{(\mu)} A_{s}^{\left(\mu^{\prime}\right)^{*}} A_{l}\right), \\
\frac{\partial A_{s}^{(\mu)}}{\partial z}+\frac{1}{v_{s}^{z(\mu)}} \frac{\partial A_{s}^{(\mu)}}{\partial t}=i \frac{2 \pi \omega_{s}}{c n_{s}^{(\mu)} \cos \theta_{s}^{z(\mu)}}\left(\chi_{s}^{\mu \sigma} A_{l} A_{p}^{(\sigma) *}+\gamma_{s}^{\mu \mu^{\prime}}\left|A_{l}\right|^{2} A_{s}^{\left(\mu^{\prime}\right)}\right), \\
2 i W^{z} \frac{\partial A_{p}^{(\sigma) *}}{\partial z}-i W e_{p}^{(\sigma) z} \frac{\partial A_{p}^{(3) *}}{\partial z}+i \frac{2 \omega_{p} \varepsilon_{p}^{(\sigma) *}}{c^{2}} \frac{\partial A_{p}^{(\sigma)^{*}}}{\partial t}+\left(W^{2}-k_{p}^{2 *}\right) A_{p}^{(\sigma) *} \\
=4 \pi q_{p}^{2} \chi_{p}^{\mu \sigma} A_{s}^{(\mu)} A_{l}^{*} \\
-i W\left(e_{p}^{(1) z} \frac{\partial A_{p}^{(1) *}}{\partial z}+e_{p}^{(2) z} \frac{\partial A_{p}^{(2) *}}{\partial z}\right)+i \frac{\mathrm{d} A_{p}^{(3) *}}{\mathrm{~d} z}\left(W^{z}-W e_{p}^{(3) z}\right) \\
+i \frac{2 \omega_{p} \varepsilon_{p}^{(3) *}}{c^{2}} \frac{\partial A_{p}^{(3) *}}{\partial t}-k_{p}^{2^{*}} A_{p}^{(3) *}=4 \pi q_{p}^{2} \chi_{p}^{\mu 3} A_{s}^{(\mu)} A_{l}^{*},
\end{gathered}
$$

Note, that in (4) $\sigma=1,2$.

Given the strong absorption we have [25]

$$
\left|W\left(A_{p}^{(\sigma)}\right)^{-1} \frac{\partial A_{p}^{(\sigma)}}{\partial z}\right| \approx\left|\frac{\omega_{p}}{c^{2}}\left(A_{p}^{(\sigma)}\right)^{-1} \frac{\partial A_{p}^{(\sigma)}}{\partial t}\right| \ll\left|W^{2}-k_{p}^{2^{*}}\right|,
$$

and we can, therefore, neglect in (6) and (7) the terms with the derivatives after which these equations yield

$$
\begin{aligned}
& A_{p}^{(\sigma)^{*}}=4 \pi \chi_{p}^{\mu \sigma} A_{s}^{(\mu)} A_{l}^{*} /\left(\mu^{2}-\varepsilon_{p}^{*}\right), \\
& A_{p}^{(3)^{*}}=-4 \pi \chi_{p}^{\mu 3} A_{s}^{(\mu)} A_{l}^{*} / \varepsilon_{p}^{*}, \mu=W / q_{p}, \sigma=1,2 .
\end{aligned}
$$

Substituting the obtained expressions in (6) and (7), we arrive at a system of two differential equations for $A_{l, s}^{(\mu)}$

$$
\begin{gathered}
\frac{\partial A_{l}}{\partial z}+\frac{1}{v_{l}^{z}} \frac{\partial A_{l}}{\partial t}=i \frac{2 \pi \omega_{l}}{c n_{l} \cos \theta_{l}^{z}}\left\{\bar{\gamma}_{l}^{\mu \sigma}\left|A_{s}^{(\mu)}\right|^{2} A_{l}+\gamma_{l}^{\mu \mu^{\prime}} A_{s}^{(\mu)} A_{s}^{\left(\mu^{\prime}\right)^{*}} A_{l}\right\}, \\
\frac{\partial A_{s}^{(\mu)}}{\partial z}+\frac{1}{v_{s}^{z(\mu)}} \frac{\partial A_{s}^{(\mu)}}{\partial t}=i \frac{2 \pi \omega_{s}}{c n_{s}^{(\mu)} \cos \theta_{s}^{z(\mu)}}\left\{\bar{\gamma}_{s}^{\mu \sigma}\left|A_{l}\right|^{2} A_{s}^{(\mu)}+\gamma_{s}^{\mu \mu^{\prime}}\left|A_{l}\right|^{2} A_{s}^{\left(\mu^{\prime}\right)}\right\},
\end{gathered}
$$

where 


$$
\begin{gathered}
\bar{\gamma}_{s}^{\mu \sigma}=4 \pi \chi_{s}^{\mu \sigma}\left(\frac{\chi_{p}^{\mu \sigma}}{\mu^{2}-\varepsilon_{p}^{*}}-\frac{\chi_{p}^{\mu 3}}{\varepsilon_{p}^{*}}\right), \bar{\gamma}_{l}^{\mu \sigma}=4 \pi \chi_{l}^{\mu \sigma}\left(\frac{\chi_{p}^{\mu \sigma}}{\mu^{2}-\varepsilon_{p}}-\frac{\chi_{p}^{\mu 3}}{\varepsilon_{p}}\right), \varepsilon_{p}=\varepsilon_{p}^{\prime}+i \varepsilon_{p}^{\prime \prime}, \\
\varepsilon_{p}^{\prime}=\varepsilon_{p}^{0}+\sum_{f} s_{f} v_{f}^{2}\left(v_{f}^{2}-v_{p}^{2}\right) /\left[\left(v_{f}^{2}-v_{p}^{2}\right)^{2}+\gamma_{f}^{2} v_{p}^{2}\right], \\
\varepsilon_{p}^{\prime \prime}=-\sum_{f} s_{f} v_{f}^{2} \gamma_{f} v_{p} /\left[\left(v_{f}^{2}-v_{p}^{2}\right)^{2}+\gamma_{f}^{2} v_{p}^{2}\right],
\end{gathered}
$$

$s_{f}$ is the oscillator strength of the o-f transition.

\section{Asymptotic Solutions: Polarization Solitons}

To do that we bring the system $(9,10)$ to unitless form.

$$
\begin{gathered}
\frac{\partial \tilde{A}_{l}}{\partial \tilde{z}}+\frac{1}{\tilde{v}_{l}^{z}} \frac{\partial \tilde{A}_{l}}{\partial \tilde{t}}=i \beta_{l}\left\{\tilde{\gamma}_{l}^{\mu \sigma}\left|\tilde{A}_{s}^{(\mu)}\right|^{2} \tilde{A}_{l}+\tilde{\gamma}_{l}^{\mu \mu^{\prime}} \tilde{A}_{s}^{(\mu)} \tilde{A}_{s}^{\left(\mu^{\prime}\right)^{*}} \tilde{A}_{l}\right\}, \\
\frac{\partial \tilde{A}_{s}^{(\mu)}}{\partial \tilde{z}}+\frac{1}{\tilde{v}_{s}^{z(\mu)}} \frac{\partial \tilde{A}_{s}^{(\mu)}}{\partial \tilde{t}}=i \beta_{s}^{(\mu)}\left\{\tilde{\gamma}_{s}^{\mu \sigma}\left|\tilde{A}_{l}\right|^{2} \tilde{A}_{s}^{(\mu)}+\tilde{\gamma}_{s}^{\mu \mu^{\prime}}\left|\tilde{A}_{l}\right|^{2} \tilde{A}_{s}^{\left(\mu^{\prime}\right)}\right\} .
\end{gathered}
$$

where $\tilde{v}_{l}^{z}=v_{l}^{z} / c, \quad \tilde{A}_{s}^{(\mu)}=A_{s}^{(\mu)} / A_{0}, \quad \tilde{A}_{l}=A_{l} / A_{0}, \quad \tilde{z}=z / z_{0}, \tilde{t}=t / \tau_{0}, \quad z_{0}=c \tau_{0}$, $\beta_{s}^{(\mu)}=2 \pi \omega_{s} z_{0} /\left(c n_{s}^{(\mu)} \cos \theta_{s}^{z(\mu)}\right), \quad \tilde{\gamma}_{s}^{\mu \sigma}=\bar{\gamma}_{s}^{\mu \sigma} A_{0}^{2}, \quad \tilde{\gamma}_{s}^{\mu \mu^{\prime}}=\gamma_{s}^{\mu \mu^{\prime}} A_{0}^{2}, \quad \tilde{v}_{s}^{z(\mu)}=v_{s}^{z(\mu)} / c$, $\beta_{l}=2 \pi \omega_{l} z_{0} /\left(c n_{l} \cos \theta_{l}^{z}\right), \quad \tilde{\gamma}_{l}^{\mu \sigma}=\bar{\gamma}_{l}^{\mu \sigma} A_{0}^{2}, \quad \tilde{\gamma}_{l}^{\mu \mu^{\prime}}=\gamma_{l}^{\mu \mu^{\prime}} A_{0}^{2}, \tau_{0}$ is the characteristic time related to the laser field (pump).

$$
\begin{aligned}
& \frac{\partial \tilde{A}_{l}}{\partial \tilde{z}}+\frac{1}{\tilde{v}_{l}^{z}} \frac{\partial \tilde{A}_{l}}{\partial \tilde{t}^{2}} \\
& =i \beta_{l}\left\{\tilde{\gamma}_{l}^{\prime \sigma}\left|\tilde{A}_{s}^{(1)}\right|^{2} \tilde{A}_{l}+\tilde{\gamma}_{l}^{2 \sigma}\left|\tilde{A}_{s}^{(2)}\right|^{2} \tilde{A}_{l}+\tilde{\gamma}_{l}^{11}\left|\tilde{A}_{s}^{(1)}\right|^{2} \tilde{A}_{l}+\tilde{\gamma}_{l}^{12} \tilde{A}_{s}^{(1)} \tilde{A}_{s}^{(2)^{*}} \tilde{A}_{l}\right. \\
& \left.\quad+\tilde{\gamma}_{l}^{21} \tilde{A}_{s}^{(2)} \tilde{A}_{s}^{(1)^{*}} \tilde{A}_{l}+\tilde{\gamma}_{l}^{22}\left|\tilde{A}_{s}^{(2)}\right|^{2} \tilde{A}_{l}\right\}, \\
& \frac{\partial \tilde{A}_{s}^{(1)}}{\partial \tilde{z}}+\frac{1}{\tilde{v}_{s}^{z(1)}} \frac{\partial \tilde{A}_{s}^{(1)}}{\partial \tilde{t}}=i \beta_{s}^{(1)}\left\{\tilde{\gamma}_{s}^{1 \sigma}\left|\tilde{A}_{l}\right|^{2} \tilde{A}_{s}^{(1)}+\tilde{\gamma}_{s}^{11}\left|\tilde{A}_{l}\right|^{2} \tilde{A}_{s}^{(1)}+\tilde{\gamma}_{s}^{12}\left|\tilde{A}_{l}\right|^{2} \tilde{A}_{s}^{(2)}\right\}, \\
& \frac{\partial \tilde{A}_{s}^{(2)}}{\partial \tilde{z}}+\frac{1}{\tilde{v}_{s}^{z(2)}} \frac{\partial \tilde{A}_{s}^{(2)}}{\partial \tilde{t}}=i \beta_{s}^{(2)}\left\{\tilde{\gamma}_{s}^{2 \sigma}\left|\tilde{A}_{l}\right|^{2} \tilde{A}_{s}^{(2)}+\tilde{\gamma}_{s}^{21}\left|\tilde{A}_{l}\right|^{2} \tilde{A}_{s}^{(1)}+\tilde{\gamma}_{s}^{22}\left|\tilde{A}_{l}\right|^{2} \tilde{A}_{s}^{(2)}\right\} .
\end{aligned}
$$

We are looking for stationary solutions as

$$
\tilde{A}_{s}^{(1,2)}(\tilde{z}, \tilde{t}) \equiv B_{s 1, s 2}(\tilde{\xi}) \mathrm{e}^{i \Phi_{s 1, s 2}(\tilde{\xi})} \text { and } \tilde{A}_{l}(\tilde{z}, \tilde{t}) \equiv B_{l}(\tilde{\xi}) \mathrm{e}^{i \Phi_{l}(\tilde{\xi})}
$$

where $\tilde{\xi} \equiv \tilde{t}-\tilde{z} / \tilde{v}^{z} ; \tilde{v}^{z}$ is the velocity of simultaneously propagating waves at the frequencies $\omega_{l, s} ; B_{l, s 1, s 2}$ and $\Phi_{l, s 1, s 2}$ are the real amplitudes and phases of the waves, respectively. Such a standard procedure of presenting the complex amplitudes of waves in terms of real and imaginary parts results in duplication of the system of (13)-(15):

$$
\begin{gathered}
\frac{\mathrm{d} B_{l}}{\mathrm{~d} \tilde{\xi}}=-\beta_{l} \tilde{\kappa}_{l}\left(\tilde{\gamma}_{l}^{12}-\tilde{\gamma}_{l}^{21}\right) B_{s 1} B_{s 2} B_{l} \sin \Phi \\
\frac{\mathrm{d} \Phi_{l}}{\mathrm{~d} \tilde{\xi}}=\beta_{l} \tilde{\kappa}_{l}\left[\left(\tilde{\gamma}_{l}^{1 \sigma}+\tilde{\gamma}_{l}^{11}\right) B_{s 1}^{2}+\left(\tilde{\gamma}_{l}^{2 \sigma}+\tilde{\gamma}_{l}^{22}\right) B_{s 2}^{2}+\left(\tilde{\gamma}_{l}^{12}+\tilde{\gamma}_{l}^{21}\right) B_{s 1} B_{s 2} \cos \Phi\right]
\end{gathered}
$$




$$
\begin{gathered}
\frac{\mathrm{d} B_{s 1}}{\mathrm{~d} \tilde{\xi}}=\beta_{s}^{(1)} \tilde{\kappa}_{s}^{(1)} \tilde{\gamma}_{s}^{12} B_{l}^{2} B_{s 2} \sin \Phi \\
\frac{\mathrm{d} B_{s 2}}{\mathrm{~d} \tilde{\xi}}=-\beta_{s}^{(2)} \tilde{\kappa}_{s}^{(2)} \tilde{\gamma}_{s}^{21} B_{l}^{2} B_{s 1} \sin \Phi \\
\frac{\mathrm{d} \Phi}{\mathrm{d} \tilde{\xi}}=\left[\beta_{s}^{(1)} \tilde{\kappa}_{s}^{(1)}\left(\tilde{\gamma}_{s}^{1 \sigma}+\tilde{\gamma}_{s}^{11}\right)-\beta_{s}^{(2)} \tilde{\boldsymbol{\kappa}}_{s}^{(2)}\left(\tilde{\gamma}_{s}^{2 \sigma}+\tilde{\gamma}_{s}^{22}\right)\right] B_{l}^{2} \\
+\left[\left(\beta_{s}^{(1)} \tilde{\boldsymbol{\kappa}}_{s}^{(1)} \tilde{\gamma}_{s}^{12} B_{l}^{2}\right) \frac{B_{s 2}}{B_{s 1}}-\left(\beta_{s}^{(2)} \tilde{\boldsymbol{\kappa}}_{s}^{(2)} \tilde{\gamma}_{s}^{21} B_{l}^{2}\right) \frac{B_{s 1}}{B_{s 2}}\right] \cos \Phi,
\end{gathered}
$$

where $\tilde{\kappa}_{l} \equiv \tilde{v}_{l} \tilde{v}^{z} /\left(\tilde{v}^{z}-\tilde{v}_{l}^{z}\right), \quad \tilde{\kappa}_{s}^{(1,2)} \equiv \tilde{v}_{s}^{z(1,2)} \tilde{v}^{z} /\left(\tilde{v}^{z}-\tilde{v}_{s}^{z(1,2)}\right), \quad \Phi \equiv \Phi_{s 1}-\Phi_{s 2}$.

We are looking for the solitary (asymptotic) solution for solitons at $\omega_{l, s}$ as following:

$$
Q=\frac{B_{l}^{2}}{\lambda_{l}^{2}}=\frac{B_{s 1}^{2}}{\lambda_{s 1}^{2}}=\frac{B_{s 2}^{2}}{\lambda_{s 2}^{2}}
$$

where $\lambda_{l}^{2} \equiv-\beta_{l} \tilde{\kappa}_{l}\left(\tilde{\gamma}_{l}^{12}-\tilde{\gamma}_{l}^{21}\right), \quad \lambda_{s 1}^{2} \equiv \beta_{s}^{(1)} \tilde{\kappa}_{s}^{(1)} \tilde{\gamma}_{s}^{12}$ and $\lambda_{s 2}^{2} \equiv-\beta_{s}^{(2)} \tilde{\kappa}_{s}^{(2)} \tilde{\gamma}_{s}^{21}$.

The introduction of $Q$ allows to reduce the system of nonlinear equations (17)-(22) to

$$
\begin{gathered}
\frac{\mathrm{d} Q}{\mathrm{~d} x}=Q^{2} \sin \Phi, \\
\frac{\mathrm{d} \Phi}{\mathrm{d} x}=Q(\tilde{m}+\tilde{n} \cos \Phi),
\end{gathered}
$$

where $x=\alpha \tilde{\xi}, \alpha \equiv 2 \lambda_{1}^{2} \lambda_{s 1} \lambda_{s 2}$,

$m \equiv\left[\beta_{s}^{(1)} \tilde{\kappa}_{s}^{(1)}\left(\tilde{\gamma}_{s}^{1 \sigma}+\tilde{\gamma}_{s}^{11}\right)-\beta_{s}^{(2)} \tilde{\kappa}_{s}^{(2)}\left(\tilde{\gamma}_{s}^{2 \sigma}+\tilde{\gamma}_{s}^{22}\right)\right] \lambda_{l}^{2}$

$n \equiv\left[\left(\beta_{s}^{(1)} \tilde{\kappa}_{s}^{(1)} \tilde{\gamma}_{s l}^{12}\right) \frac{\lambda_{s 2}}{\lambda_{s 1}}-\left(\beta_{s}^{(2)} \tilde{\kappa}_{s}^{(2)} \tilde{\gamma}_{s l}^{21}\right) \frac{\lambda_{s 1}}{\lambda_{s 2}}\right] \lambda_{l}^{2}, \quad \tilde{m} \equiv m / \alpha \quad, \quad \tilde{n} \equiv n / \alpha \quad . \quad$ The space-time evolution of the normalized intensities is shown in Figure 1.

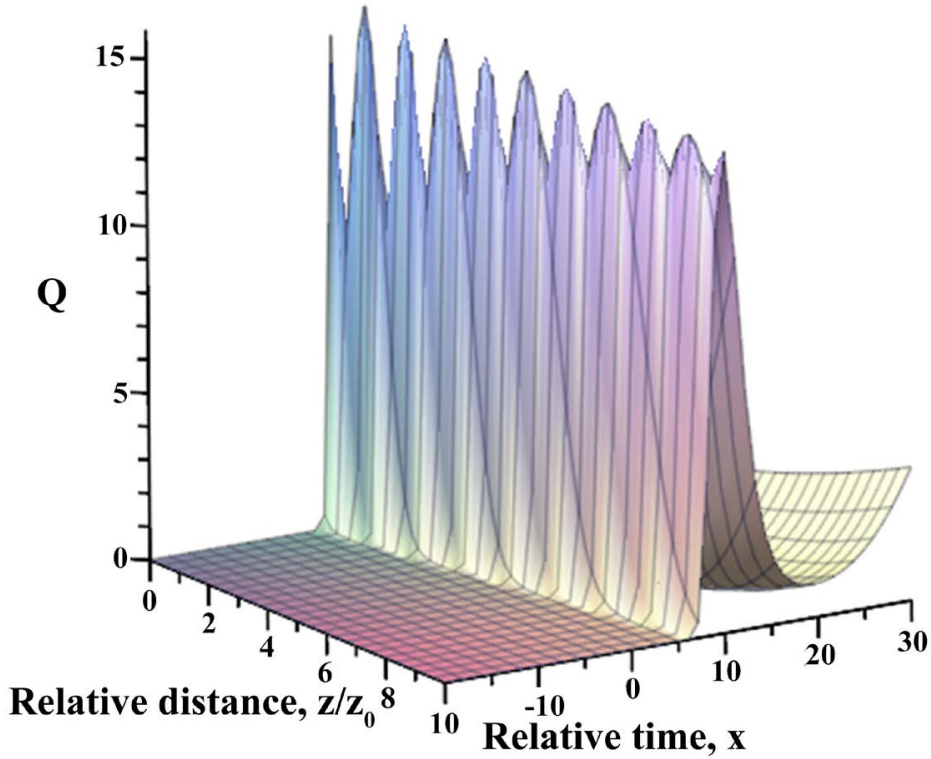

(a) 


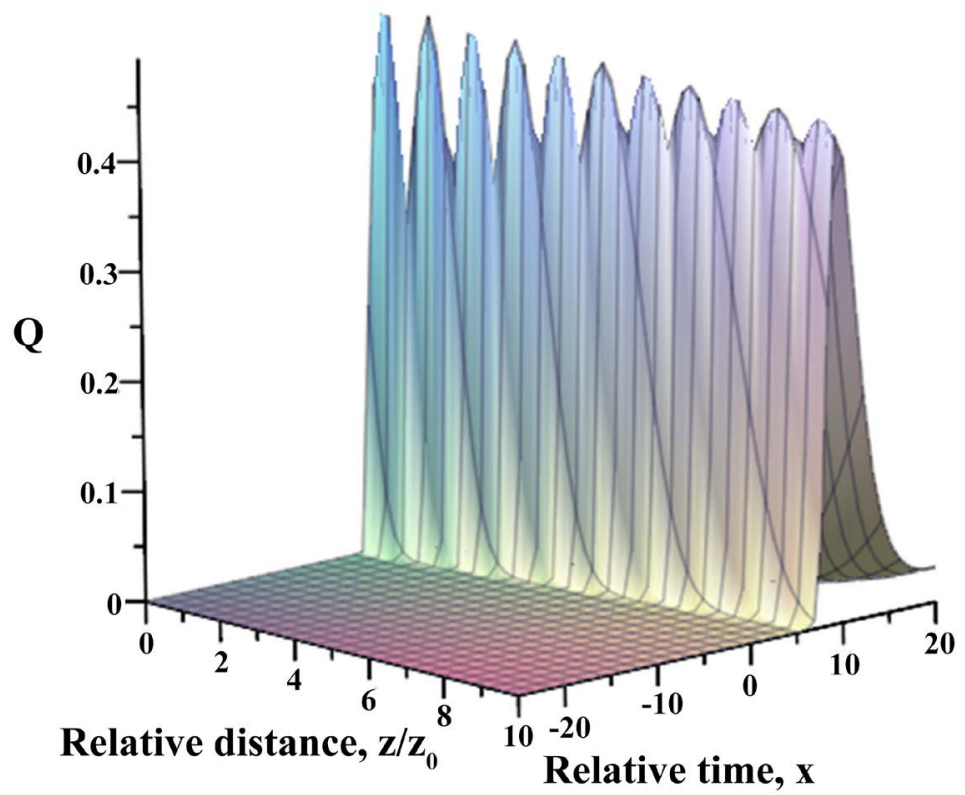

(b)

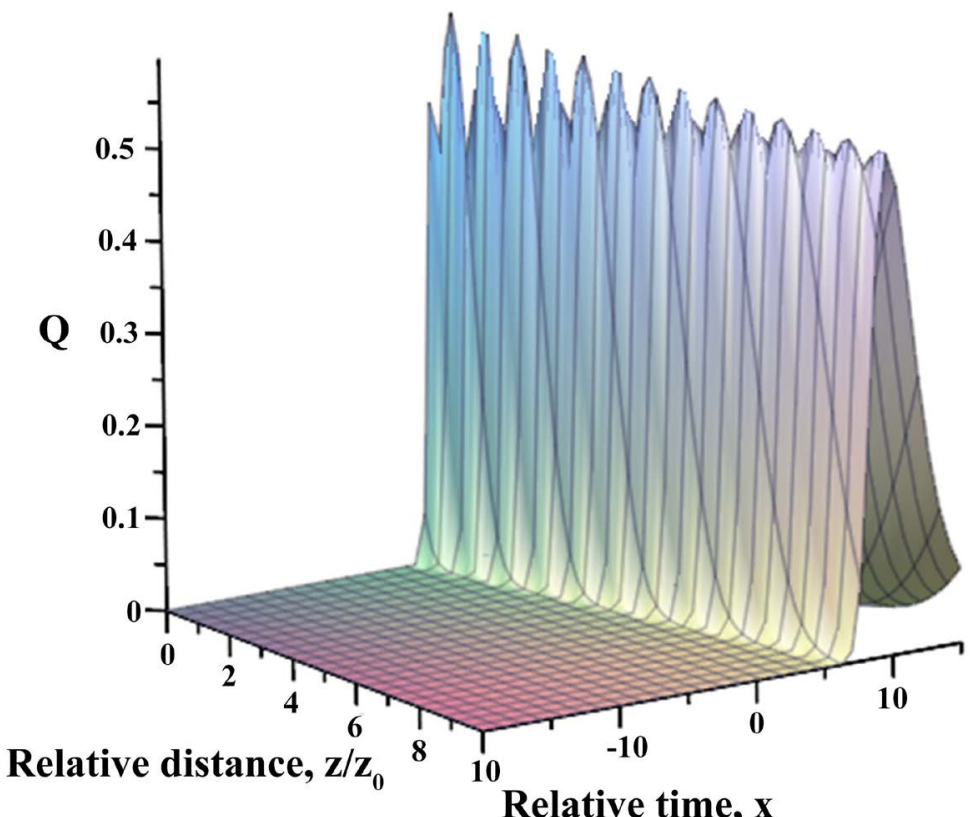

(c)

Figure 1. The space-time evolution of the normalized intensities of the pump and Stokes pulses for: (a) $\tilde{m}=1.0$ and $\tilde{n}=1.0$, (b) $\tilde{m}=1.0$ and $\tilde{n}=0.01$, and (c) $\tilde{m}=1.0$ and $\tilde{n}=0.1$, respectively.

\section{Gain Factor $g_{\mu}$}

Now we show that the system of Equations (14) and (15) is consistent with the experimental results presented, for example, in [27]. To do that we first bring the system of Equations (14) and (15) to unitless form and change the variables $z, t$ to variables $\tilde{z}, \tilde{\tau}=\tilde{t}-\tilde{z} / \tilde{v}_{s}^{z} \quad$ (we assume that $\tilde{v}_{s}^{z(1)} \approx \tilde{v}_{s}^{z(2)} \approx \tilde{v}_{s}^{z}$ and consider 
the preset-pump approximation):

$$
\frac{\partial \tilde{A}_{s}^{(\mu)}}{\partial \tilde{z}}=i \beta_{s}^{(\mu)}\left\{\tilde{\gamma}_{s}^{\mu \sigma}\left|\tilde{A}_{l}\right|^{2} \tilde{A}_{s}^{(\mu)}+\tilde{\gamma}_{s}^{\mu \mu^{\prime}}\left|\tilde{A}_{l}\right|^{2} \tilde{A}_{s}^{\left(\mu^{\prime}\right)}\right\},
$$

where $\tilde{A}_{s}^{(\mu)}=A_{s}^{(\mu)} / A_{0}, \quad \tilde{A}_{l}=A_{l} / A_{0}, \quad \tilde{z}=z / z_{0}, \tilde{t}=t / \tau_{0}, \quad z_{0}=c \tau_{0}$, $\beta_{s}^{(\mu)}=2 \pi \omega_{s} z_{0} /\left(c n_{s}^{(\mu)} \cos \Theta_{s}^{z(\mu)}\right), \quad \tilde{\gamma}_{s}^{\mu \sigma}=\bar{\gamma}_{s}^{\mu \sigma} A_{0}^{2}, \quad \tilde{\gamma}_{s}^{\mu \mu^{\prime}}=\gamma_{s}^{\mu \mu^{\prime}} A_{0}^{2}, \quad \tilde{v}_{s}^{z(\mu)}=v_{s}^{z(\mu)} / c$, $\tau_{0}$ is the characteristic time related to the laser field (pump).

The theoretical consideration of the gain factor for SRS by polaritons is based on the modeling of the quasi-stationary solutions of the coupled wave equations for the different polarizations of the Stokes (for the complete analysis see [25]). Therefore, we seek the solutions of (25) in the form $\tilde{A}_{s}^{(\mu)}=B_{\mu} \exp (\kappa \tilde{z})$, assuming $B_{\mu}$ and $\kappa$ to be independent of $\mathrm{z}$. We then obtain the system of algebraic equations for $B_{\mu}$. Choosing in a plane perpendicular to a two-dimensional coordinate system with axes along the unit vectors, we represent the equations for $B_{\mu}$ in the form of a tensor relation

$$
\Delta_{\mu \mu^{\prime}} B_{\mu^{\prime}}=-i \kappa B_{\mu}, \quad \mu=1,2
$$

where $\Delta_{\mu \mu^{\prime}}=\beta_{s}^{(\mu)}\left|\tilde{A}_{l}\right|^{2}\left(\tilde{\gamma}_{s}^{\mu \mu^{\prime}}+\tilde{\gamma}_{s}^{\mu \sigma}\right)$.

Equating the determinant of the system (26) to zero, we obtain the solutions for $\kappa$

$$
\kappa=i\left[\left(\Delta_{11}+\Delta_{22}\right) \pm \sqrt{\left(\Delta_{11}-\Delta_{22}\right)^{2}+4 \Delta_{12} \Delta_{21}}\right] / 2 .
$$

We will need the explicit expressions for the tensors $\chi$ and $\gamma$. They can be found within the framework of the microscopic theory in the dipole approximation based on the perturbation theory states [25]. The resultant expressions are

$$
\begin{gathered}
\chi_{i j k}\left(\omega_{l},-\omega_{p}\right)=\chi_{i j k}^{\circ}\left(\omega_{l},-\omega_{p}\right)+\frac{\sqrt{N}}{\hbar} \sum_{f v} \alpha_{i j}^{(f v)} P_{f v}^{k} F_{f}\left(\omega_{p}\right) \\
\gamma_{i j k m}=\frac{1}{\hbar v_{0}} \sum_{f v} \alpha_{i k}^{(f v)} \alpha_{j m}^{(f v)} F_{f}\left(\omega_{p}\right)+\gamma_{i j k m}^{\circ},
\end{gathered}
$$

where $F_{f}(\omega) \approx 2 \omega_{t} /\left(\omega_{f}^{2}-\omega_{p}^{2}+i \tilde{\gamma} \omega_{p}\right)$.

The summation in (28) and (29) is over all dipole-active phonons, the frequencies of which are equal $\omega_{f}-i \tilde{\gamma}_{f} / 2$, where $\tilde{\gamma}_{f}$ are the attenuation constants. For example in a cubic crystal, the dipole-active phonons are triply degenerate [25] so that the number of the mutually degenerated oscillations we introduce the index $v \quad\left(\boldsymbol{e}_{v}\right.$ is a triad of real unit vectors denoting the vibrations along the edges of the unit cube. Furthermore, $\boldsymbol{P}_{f v}=P_{f} \boldsymbol{e}_{v}$ is the dipole moment of the transition $0-\mathrm{fv}$ for the unit cell with its volume $v_{0} ; \alpha_{i j}^{(f v)}$ is the tensor of the phonon spontaneous scattering per cell [28]; $N=V / v_{0}$ is the number of cells in the crystal. The tensor $\chi_{i j k}^{\circ}$ represents the contribution to $\chi_{i j k}$ the remote electronic states. The tensor $\gamma_{i j k m}^{\circ}$ determines the contribution due to the electronic states as well. It is convenient to represent the tensors $\chi_{i j k}(28)$ and $\gamma_{i j k m}$ (29) in the simplified form as follows 


$$
\begin{aligned}
& \chi_{i j k}=\chi_{i j k}^{\prime}+i \chi_{i j k}^{\prime \prime}, \chi_{i j k}^{\prime}=\chi_{i j k}^{0}+\sum_{f} \chi_{f} v_{f}^{2}\left(v_{f}^{2}-v_{p}^{2}\right) /\left[\left(v_{f}^{2}-v_{p}^{2}\right)^{2}+\gamma_{f}^{2} v_{p}^{2}\right], \\
& \chi_{i j k}^{\prime \prime}=-\sum_{f} \chi_{f} v_{f}^{2} \gamma_{f} v_{p} /\left[\left(v_{f}^{2}-v_{p}^{2}\right)^{2}+\gamma_{f}^{2} v_{p}^{2}\right], \\
& \gamma_{i j k}=\gamma_{i j k}^{\prime}+i \gamma_{i j k}^{\prime \prime}, \gamma_{i j k}^{\prime}=\gamma_{i j k}^{0}+\sum_{f} \gamma^{f} v_{f}^{2}\left(v_{f}^{2}-v_{p}^{2}\right) /\left[\left(v_{f}^{2}-v_{p}^{2}\right)^{2}+\gamma_{f}^{2} v_{p}^{2}\right], \\
& \gamma_{i j k m}^{\prime \prime}=-\sum_{f} \gamma^{f} v_{f}^{2} \gamma_{f} v_{p} /\left[\left(v_{f}^{2}-v_{p}^{2}\right)^{2}+\gamma_{f}^{2} v_{p}^{2}\right],
\end{aligned}
$$

where $\chi_{f}=(h c / 2 \pi)^{-1 / 2} v_{l}^{-2}\left(s_{f} \sigma_{f} / v_{f}\right)^{1 / 2}, \quad \gamma^{f}=\left(8 \pi^{2} \sigma_{f}\right) /\left(h c v_{l}^{4} v_{f}\right), \quad \sigma_{f}$ is the Raman differential cross-section per unit cell $v_{0}\left(\mathrm{~cm}^{-1} / \mathrm{sr}\right)$.

We introduce the principal axes of the tensor $\Delta_{\mu \mu 1}$ as a whole. If we denote its principal values as $\Delta_{\mu}$ we obtain from (26) $\kappa_{\mu}=i \Delta_{\mu}$. Finally, we introduce the gain $g_{\mu}=2 R e \kappa_{\mu}$ which can be expressed as

$$
g_{\mu}=\frac{8 \pi^{2} \omega_{l} \omega_{s} z_{0} I_{l}}{c^{2} n_{l} n_{s} \cos \theta}\left(4 \pi\left[\frac{K_{\mu}^{\prime} \varepsilon_{p}^{\prime \prime}-K_{\mu}^{\prime \prime}\left(s^{2}-\varepsilon_{p}^{\prime}\right)}{\left(s^{2}-\varepsilon_{p}^{\prime}\right)^{2}+\varepsilon_{p}^{\prime 2}}+\frac{L_{\mu}^{\prime} \varepsilon_{p}^{\prime \prime}+L_{\mu}^{\prime \prime} \varepsilon_{p}^{\prime}}{\varepsilon_{p}^{\prime 2}+\varepsilon_{p}^{\prime \prime 2}}\right]-M_{\mu}\right), \mu=1,2
$$

where $K_{\mu}=\sum\left(\chi^{\mu \sigma}\right)^{2}=K_{\mu}^{\prime}+i K_{\mu}^{\prime \prime}, \quad L_{\mu}=\left(\chi^{\mu 3}\right)^{2}=L_{\mu}^{\prime}+i L_{\mu}^{\prime \prime}, \quad I_{l}=c n_{l}\left|A_{l}\right|^{2} / 2 \pi$ is the pump intensity, $M_{\mu}$ are the principal values of the tensor $\gamma_{s}^{\left(\mu \mu^{\prime}\right)^{\prime \prime}}, \theta$ is the scattering angle (the angle between $\boldsymbol{k}_{l}$ and $\boldsymbol{k}_{s}\left(n_{s}^{(\mu)} \approx n_{s}, \cos \theta_{s}^{z(\mu)} \simeq \cos \theta\right)$ ).

Formula (31) denotes two gain coefficients for Stokes waves polarized along $\boldsymbol{e}_{s}^{(\mu)}$.

To verify (31), we were using the parameters of crystals widely used in optical display and storage, optical communication network, optical detection, etc. such as $\mathrm{ZnO}$ [29]-[35] and $\mathrm{ZnS}$ [36] [37] [38] [39]. Figure 2 shows the intensity as a function of the polariton frequency in zinc blende $\mathrm{ZnS}$ in the range $200-400$ $\mathrm{cm}^{-1}$. The red dots represent the experimental points [27].

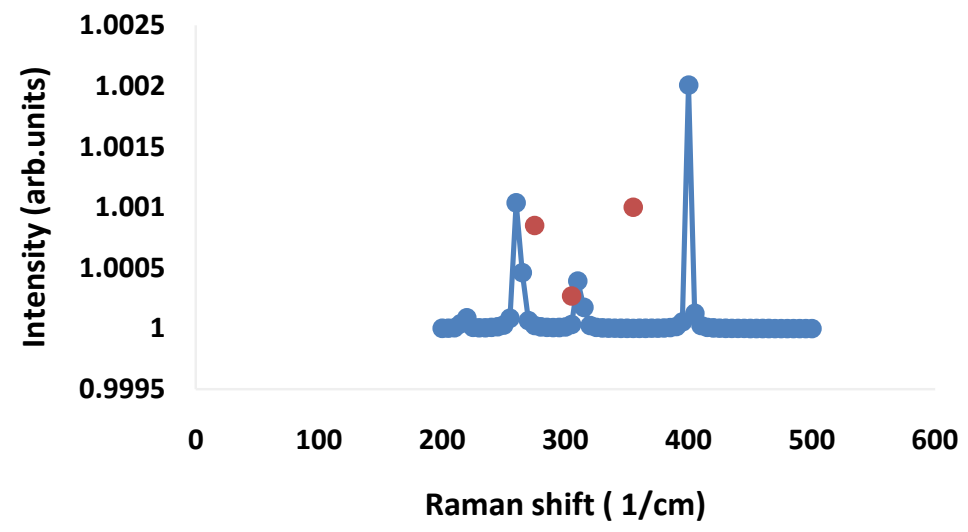

Figure 2. The graph of intensity (in arbitrary units) versus the polariton frequency (in $1 / \mathrm{cm}$ ) in $\mathrm{ZnS}$ in the range $200-4001 / \mathrm{cm}$.

\section{Conclusion}

In this paper, we theoretically showed that in the case of nonstationary SRS by polaritons, there is a possibility of occurrence of simultaneously propagating ul- 
trafast stable pulses (simultons) not only at different frequencies but with different polarizations as well. This can be used in optoelectronics when creating polarization filters.

\section{Conflicts of Interest}

The authors declare no conflicts of interest regarding the publication of this paper.

\section{References}

[1] Rosas, A.F., Hernández, O.D., Arceo, R., Santos, G.J.E., Vázquez, S.M., Álvarez, E.R., Flores, C.I.E. and Kuzin, E. (2018) Polarization Properties, Nonlinear Optics-Novel Results in Theory and Application.

[2] Millot, G., TchofoDinda, P., Seve, E. and Wabnitz, S. (2001) Modulational Instability and Stimulated Raman Scattering in Normally Dispersive Highly Birefringent Fibers. Optical Fiber Technology, 7, 170-205. https://doi.org/10.1006/ofte.2000.0356

[3] Cheng, T.L., Wang, Q.M., Yan, X., Wang, F., Zhang, X.N., Li, S.G., Suzuki, T. and Ohishi, Y. (2020) Experimental Investigation of the Polarization Modulation Instability and Stimulated Raman Scattering in a Chalcogenide Optical Fiber. Journal of Applied Physics, 128, Article ID: 193103. https://doi.org/10.1063/5.0022957

[4] Flores-Rosas, A., Kuzin Evgeny, A., Arceo, R., Díaz-Hernán, O., Ruiz-Pérez, V.I. and Escalera-Santos, G.J. (2017) Polarization Properties of Vector Solitons Generated by Modulation Instability in Fiber with Circular Birefringence. Optical Engineering, 56, Article ID: 036115. https://doi.org/10.1117/1.OE.56.3.036115

[5] Ortiz-Mora, A., Rodríguez, P., Díaz-Soriano, A., Martínez-Muñoz, D. and Dengra, A. (2020) Method of Moments Optimization of Distributed Raman Amplification in Fibers with Randomly Varying Birefringence. Photonics, 7, 86. https://doi.org/10.3390/photonics7040086

[6] Kaplan, A.E., Shkolnikov, P.L. and Akanaev, B.A. (1994) Bright-Bright $2 \pi$ Solitons in Stimulated Raman Scattering. Optics Letters, 19, 445-447. https://doi.org/10.1364/OL.19.000445

[7] Boiti, M., Caputo, J.-G., Leon, J. and Pempinelli, F. (2000) Raman Solitons in Transient SRS. Inverse Problems, 16, 303. https://doi.org/10.1088/0266-5611/16/2/303

[8] Kalashnikov, V.L. and Sorokin, E. (2014) Dissipative Raman Solitons. Optics EXpress, 22, 30118-30126. https://doi.org/10.1364/OE.22.030118

[9] Karpov, M., Pfeiffer Martin, H.P., Guo, H.R., Weng, W.L., Liu, J.Q. and Kippenberg, T.J. (2019) Dynamics of Soliton Crystals in Optical Micro-Resonators. Nature Physics, 15, 1071-1077.

[10] Frank, M., Smetanin, S.N., Jelinek, M., Vyhlidal, D., Shukshin, V.E., Ivleva, L.I., Dunaeva, E.E., Voronina, I.S., Zverev, P.G. and Kubecek, V. (2019) Stimulated Raman Scattering in Alkali-Earth Tungstate and Molybdate Crystals at Both Stretching and Bending Raman Modes under Synchronous Picosecond Pumping with Multiple Pulse Shortening Down to 1 ps. Crystals, 9, 167.

https://doi.org/10.3390/cryst9030167

[11] Hill, A.H., Munger, E., Francis, A.T., Manifold, B. and Fu, D. (2019) Frequency Modulation Stimulated Raman Scattering Microscopy through Polarization Encoding. The Journal of Physical Chemistry B, 123, 8397-8404.

https://doi.org/10.1021/acs.jpcb.9b07075 
[12] Chen, T., Yavuz, A. and Wang, M.C. (2021) Dissecting Lipid Droplet Biology with Coherent Raman Scattering Microscopy. Journal of Cell Science, 135, No. 5. https://doi.org/10.1242/jcs.252353

[13] Xiong, H.Q., Qian, N.X., Miao, Y.P., Zhao, Z.L., Chen, C. and Min, W. (2021) Super-Resolution Vibrational Microscopy by Stimulated Raman Excited Fluorescence. Light: Science \& Applications, 10, Article No. 87. https://doi.org/10.1038/s41377-021-00518-5

[14] Li, Y.P., Shen, B.L., Zou, G.J., Wang, S.Q., Qu, J.L., Hu, R. and Liu, L.W. (2021) Fast Denoising and Lossless Spectrum Extraction in Stimulated Raman Scattering Microscopy. Journal of Biophotonics, 14, e202100080.

[15] Jiang, J., Grass, D., Zhou, Y., Warren, W.S. and Fischer, M.C. (2021) Beyond Intensity Modulation: New Approaches to Pump-Probe Microscopy. Optics Letters, 46, 1474. https://doi.org/10.1364/OL.417905

[16] Lee, M., Herrington, C.S., Ravindra, M., Sepp, K., Davies, A., Hulme, A.N. and Brunton, V.G. (2021) Recent Advances in the Use of Stimulated Raman Scattering in Histopathology. The Analyst, 146, 789-802.

https://doi.org/10.1039/D0AN01972K

[17] Xiong, H.Q., Qian, N.X., Zhao, Z.L., Shi, L.Y., Miao, Y.P. and Min, W. (2020) Background-Free Imaging of Chemical Bonds by a Simple and Robust Frequency-Modulated Stimulated Raman Scattering Microscopy. Optics Express, 28, 15663-15677. https://doi.org/10.1364/OE.391016

[18] Dong, P.-T., Zong, C., Dagher, Z., Hui, J., Li, J.J., Zhan, Y.W., Zhang, M., Mansour, M.K. and Cheng, J.-X. (2021) Polarization-Sensitive Stimulated Raman Scattering Imaging Resolves Amphotericin B Orientation in Candida Membrane. Science Advances, 7, eabd5230. https://doi.org/10.1126/sciadv.abd5230

[19] Angelakis, D.G. (2017) Quantum Simulations with Photons and Polaritons. Springer, Berlin. https://doi.org/10.1007/978-3-319-52025-4

[20] Burstein, E., Ushioda, S. and Pinczuk, A. (1968) Raman Scattering by Polaritons. Solid State Communications, 6, 407-411. https://doi.org/10.1016/0038-1098(68)90168-3

[21] Claus, R., Merten, 1. and Brandmuller, J. (1975) Light Scattering by Phonon-Polaritons. Springer, Berlin.

[22] Agranovich, V. (1982) Surface Polaritons. Elsevier, Amsterdam. https://doi.org/10.1016/B978-0-444-86165-8.50006-2

[23] Cottam, M.G. and Tilley, D.R. (2019) Introduction to Surface and Superlattice Excitations. Taylor Francis Group, Bristol and Philadelphia. https://doi.org/10.1201/9780429187049

[24] Boyd, R. (2019) Nonlinear Optics. 4th Edition, Elsevier, Amsterdam.

[25] Strizhevskii, V.L. (1972) Theory of Stimulated Raman Scattering by Polaritons in Cubic and Uniaxial Crystals. Journal of Experimental and Theoretical Physics (JETP), 35, 760-766.

[26] Akhmanov, S.A. and Khokhlov, R.V. (1964) Problems of Nonlinear Optics.

[27] Cheng, Y.C., Jin, C.Q., Gao, F., Wu, X.L., Zhong, W., Li, S.H. and Chu, P.K. (2009) Raman Scattering Study of Zinc-Blende and Wurtzite ZnS. Journal of Applied Physics, 106, Article ID: 123505. https://doi.org/10.1063/1.3270401

[28] Strizhevskii, V.L. (1962) Raman Scattering of Light in Crystals. Physics of the Solid State, 3, 2929.

[29] Fonoberov, V.A. and Balandin, A.A. (2004) Interface and Confined Polar Optical 
Phonons in Spherical ZnO Quantum Dots with a Wurtzite Crystal Structure. Physica Status Solidi (c), 1, 2650-2653. https://doi.org/10.1002/pssc.200405373

[30] Alim, K.A., Fonoberov, V.A. and Balandin, A.A. (2005) Origin of the Optical Phonon Frequency Shifts in ZnO Quantum Dots. Applied Physics Letters, 86, Article ID: 053103. https://doi.org/10.1063/1.1861509

[31] Serrano, J., Manjon, E.J., Romero, A.H., Ivanov, A. Cardona, M., Lauck, R., Bosak, A. and Krisch, M. (2010) Phonon Dispersion Relations of Zinc Oxide: Inelastic Neutron Scattering and ab Initio Calculations. Physical Review B, 81, Article ID: 174304. https://doi.org/10.1103/PhysRevB.81.174304

[32] Bhunia, A.K., Jha, P.K., Rout, D. and Saha, S. (2016) Morphological Properties and Raman Spectroscopy of ZnO Nanorods. Journal of Physical Sciences, 21, 111-118.

[33] Phan, T.L., Yu, S.C., Nghia, N.X. and Lam, V.D. (2010) Resonant Raman Scattering in $\mathrm{ZnO}$ Nanostructures Annealed at Different Temperatures. Journal of the Korean Physical Society, 57, 1569-1573. https://doi.org/10.3938/jkps.57.1569

[34] Mallet, E., Reveret, F., Disseix, P., Shubina, T.V. and Leymarie, J. (2014) Influence of Excitonic Oscillator Strengths on the Optical Properties of GaN and ZnO. Physical Review B, 90, Article ID: 045204. https://doi.org/10.1103/PhysRevB.90.045204

[35] Milekhin, A.G., Yerykov, N.A., Sveshnikova, L.L., Duda, T.A., Zenkevitch, E.I., Kosolobov, S.S., Latyshev, A.V., Himenski, C., Surovtsev, N.V., Adichchev, S.V., Feng, Z.C., Wu, C.C., Wuu, D.S. and Zahn, D.R.T. (2011) Surface-Enhanced Raman Scattering of Light by $\mathrm{ZnO}$ Nanostructures. Journal of Experimental and Theoretical Physics, 113, 983-991. https://doi.org/10.1134/S1063776111140184

[36] Zeng, X., Yan, S., Cui, J., Liu, H., Dong, J., Xia, W., Zhou, M. and Chen, H. (2015) Size- and Morphology-Dependent Optical Properties of ZnS: Al One-Dimensional Structures. Journal of Nanoparticle Research, 17, 188. https://doi.org/10.1007/s11051-015-3000-y

[37] Serrano, J., Cantarero, A., Cardona, M., Garro, N., Lauck, R., Tallman, R.F., Ritter, T.M. and Weinstein, B.A. (2004) Raman Scattering in $\beta$-ZnS. Physical Review B, 69, Article ID: 014301. https://doi.org/10.1103/PhysRevB.69.014301

[38] Vagelatos, N., Wehe, D. and King, J.S. (1974) Phonon Dispersion and Phonon Densities of States for ZnS and ZnTe. The Journal of Chemical Physics, 60, 3613-3618. https://doi.org/10.1063/1.1681581

[39] Sun, L., Shi, L.C. and Wang, C.R. (2016) Investigations of Phonons in Zinc-Blende and Wurtzite by Raman Spectroscopy. IntechOpen, London, 23-40.

https://doi.org/10.5772/64194 